\title{
Camtracker: a new camera controlled high precision solar tracker system for FTIR-spectrometers
}

\author{
M. Gisi, F. Hase, S. Dohe, and T. Blumenstock \\ Karlsruhe Institute of Technology (KIT), Institute for Meteorology and Climate Research (IMK-ASF), Karlsruhe, Germany \\ Received: 29 October 2010 - Published in Atmos. Meas. Tech. Discuss.: 9 November 2010 \\ Revised: 14 January 2011 - Accepted: 17 January 2011 - Published: 19 January 2011
}

\begin{abstract}
A new system to very precisely couple radiation of a moving source into a Fourier Transform Infrared (FTIR) Spectrometer is presented. The Camtracker consists of a homemade altazimuthal solar tracker, a digital camera and a homemade program to process the camera data and to control the motion of the tracker. The key idea is to evaluate the image of the radiation source on the entrance field stop of the spectrometer. We prove that the system reaches tracking accuracies of about 10 arc $\mathrm{s}$ for a ground-based solar absorption FTIR spectrometer, which is significantly better than current solar trackers. Moreover, due to the incorporation of a camera, the new system allows to document residual pointing errors and to point onto the solar disk center even in case of variable intensity distributions across the source due to cirrus or haze.
\end{abstract}

\section{Introduction}

Ground-based solar absorption Fourier Transform Infrared (FTIR) measurements are performed at numerous sites worldwide to monitor trace gases in the terrestrial atmosphere. Prominent FTIR networks are the "Network for the Detection of Atmospheric Composition Change" (NDACC, http://www.ndsc.ncep.noaa.gov), operating in the mid-infrared (MIR) spectral region and encompassing over 20 measurement sites worldwide and the "Total Carbon Column Observing Network" (TCCON, http://www.tccon. caltech.edu), focusing mainly on $\mathrm{CO}_{2}$ measurements in the near infrared (NIR) at about 20 sites worldwide (Toon et al., 2009; Wunch et al., 2010a,b). As one aims to increase the

Correspondence to: $\mathrm{M}$. Gisi (michael.gisi@kit.edu) accuracy of the retrieved column-averaged abundances of atmospheric constituents, new challenges arise.

In the past, major efforts were undertaken to improve the spectrometer itself, such as the ILS-characterization (Hase et al., 1999), applying a DC-correction on the interferogram (Keppel-Aleks et al., 2007), improving the sampling accuracy (Messerschmidt et al., 2010) and characterization of detector non-linearities (Abrams et al., 1994). But in order to reach the requested accuracy, the tracking quality also has to be considered, which is still an open issue. The current approach to reach an optimal tracking precision is to use a quadrant-diode to register deviations from the precalculated pointing direction of the tracking system. The diode signal is then fed in the control loop of the tracker (Adrian et al., 1994; Notholt and Schrems, 1995; Washenfelder et al., 2006). However, these systems do not achieve the required accuracy in some conditions, especially at low solar elevation. In addition they are prone to misalignments between the line-of-sight (LOS) defined by the quadrant sensor and the LOS of the spectrometer which cause systematic and noncorrectable errors. They also require strict conditions on the shape and intensity distribution of the light source. We have overcome these problems by controlling the tracking with a new camera-based system.

\section{Importance of the tracking accuracy}

When using the sun as a light source for atmospheric absorption spectroscopy, one usually aims to point the interferometer's field-of-view on the center of the solar disk equivalent to centering the solar disk on the circular entrance field stop of the interferometer. Any deviations from the assumed LOS introduce errors in the retrieval of the gas concentrations of the atmosphere. The main problem caused by a pointing error

Published by Copernicus Publications on behalf of the European Geosciences Union. 


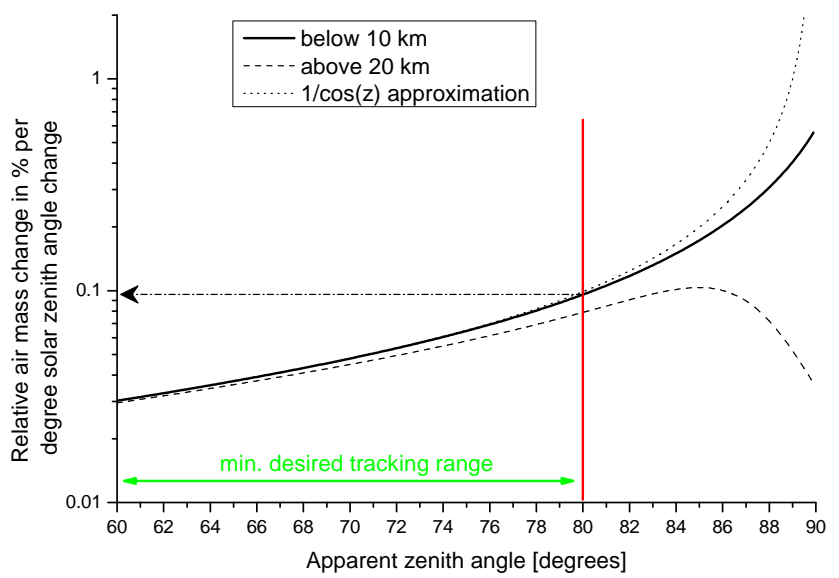

Fig. 1. Sensitivity of the effective air mass as function of solar zenith angle for the observation of a tropospheric gas with constant VMR up to $10 \mathrm{~km}$ (bold line), for the observation of a stratospheric gas with constant VMR above $20 \mathrm{~km}$ (dashed) and for the often used analytical approximation $1 / \cos (\mathrm{z})$ (dotted). (Graph taken from Hase, 2000.)

is that the actual observed air mass differs from the air mass assumed in the analysis. The error resulting from a line-ofsight (LOS) error depends strongly on the zenith angle of the sun and is shown in Fig. 1. With a desired tracking range from $0^{\circ}$ to $80^{\circ}$ solar zenith angle (SZA), one gets up to $9.6 \%$ air mass change per degree SZA change. TCCON currently strives for a total $\mathrm{CO}_{2}$ column precision of $0.1 \%$ in order to constrain the interhemispheric gradient (Olsen and Randerson, 2004). To achieve this overall precision, an error in the total gas column due to the tracking of smaller than $0.05 \%$ is desirable. If one wants to maintain this for a tropospheric gas up to a solar zenith angle of $80^{\circ}$, a tracking accuracy of about 19 arc s is required.

Although it is possible to reduce the tracker impact in the NIR by ratioing the $\mathrm{CO}_{2}$ retrieved slant column to the $\mathrm{O}_{2}$ retrieved slant column from the same spectrum, an excellent tracking knowledge is nevertheless highly desirable, because this allows to recognize other problems by monitoring the observed $\mathrm{O}_{2}$ column. Moreover, in the MIR spectral region, no reference of similar precision is available, so the calibration using $\mathrm{O}_{2}$ is not feasible in case of NDACC measurements.

\section{Tracking accuracy with current quadrant-diode setups}

An additional effect of mispointing of the solar tracker is to generate a Doppler shift of the solar lines with respect to the telluric spectral features due to the solar rotation. The synodic rotation period of the sun is about 26.75 days, which corresponds to an observed equatorial solar velocity of about $1890 \mathrm{~m} \mathrm{~s}^{-1}$ (Lang, 1991). A mispointing along the solar equator of 1 arc min translates into a Doppler scaling $\Delta v / v$ of $3.9 \times 10^{-7}$. If this effect is considered in the analysis by fitting a separate shift for the solar background lines, the effects on the trace gas analysis are minor, but it gives a useful method to estimate the pointing quality at hand. Note, however, that the mispointing cannot be retrieved from the observed Doppler shifts, because there is no sensitivity along the direction parallel to the solar rotation axis. For this reason, we apply in the following an additional factor of $\sqrt{2}$ when we estimate the total pointing error (we assume that the pointing uncertainty is of the same size for any direction on the solar disk).

For the analysis we used the software PROFFIT Ver. 9.6 which was developed at IMK (Hase et al., 2004). A model of the solar absorption lines is included in this code (Hase et al., 2006) and (Hase et al., 2010). The solar line list for the NIRregion was provided by $\mathrm{G}$. Toon, JPL (G. Toon, personal communication, 2004). To estimate current tracking accuracies with a quadrant diode setup, we evaluated the spectra measured at our FTIR sites Kiruna $\left(67.84^{\circ} \mathrm{N}, 20.41^{\circ} \mathrm{E}\right)$ and Izaña $\left(28.30^{\circ} \mathrm{N}, 16.48^{\circ} \mathrm{W}\right)$.

The solar trackers at the two sites are not fully identical. In Kiruna, the solar light that is analyzed by the quadrant diode is decoupled from the converging beam a few $\mathrm{cm}$ in front of the entrance aperture by a tiny plane mirror, so only a small subsection of the full beam diameter is used. In Izaña, a plane semitransparent mirror is inserted between the spectrometer's focusing parabolic mirror and the entrance aperture, which covers the full beam diameter and reflects the IR radiation towards the spectrometer while a fraction of the VIS radiation is transmitted towards the quadrant sensor. We believe that the Kiruna setup is representative for most FTIR solar trackers in operation, whereas the Izaña setup might be the optimum which can be achieved with the quadrant diode approach, because the same beam is used by the quadrant sensor as by the spectrometer. This solution is insensitive against optical aberrations of the off-axis paraboloid and has to our knowledge not been realized at other FTIR sites so far.

The deduced pointing accuracy for Kiruna is shown in Fig. 2, indicating a tracking accuracy of $\sqrt{2} \times \pm 69$ arc s $\approx \pm 98$ arc s. Note that any realignment of the quadrant sensor tends to affect the quality of the tracking, this is probably the reason for the reduced scatter since Febuary 2006. At the FTIR site Izaña, we installed the setup with the semitransparent mirror in February 2005 and observed an accuracy of 93 arc s. After small realignments of the quadrant diode in May 2007 we reached 34 arc s.

Even the final accuracies reported above are not sufficient for the desired gas retrieval precision of $0.1 \%$, as these values correspond to an air mass change of about $0.3 \%$ (Kiruna), respectivey $0.09 \%$ (Izaña) at a solar zenith angle of $80^{\circ}$. 


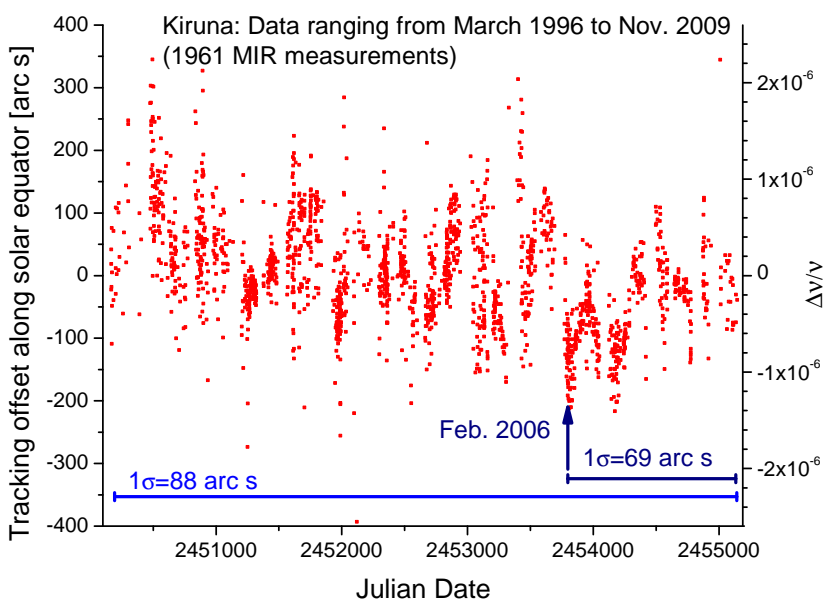

Fig. 2. Tracking angle offset determined by solar shifts for the measurement site in Kiruna as a typical NDACC station. For both directions, a tracking accuracy of $\pm 98 \operatorname{arcs~}(1 \sigma)$ can be estimated for the time after February 2006. The evaluated spectral window ranged from 2703.2 to 2705.3 wavenumbers.

\section{Camtracker setup}

The demonstration setup is located at our measurement site near Karlsruhe, Germany $\left(49.10^{\circ} \mathrm{N}, 8.44^{\circ} \mathrm{E}\right)$. We use a $20 \mathrm{ft}$ container to house our IFS 125HR FTIR-spectrometer from Bruker Optics $\mathrm{GmbH}$, with a maximal optical path difference $(\mathrm{OPD})_{\max }$ of $257 \mathrm{~cm}$. This is equipped with a $\mathrm{CaF}_{2}$ beamsplitter, an InSb and an InGaAs-detector. The tracker is mounted on top of the container, and has, except for the motor-types, the same technical setup as our trackers in Izaña and Kiruna, which have been operational since 1999 and 1998, respectively. The mechanical setup of the altazimuthal tracker is shown in Fig. 3. The sunlight is reflected into the container by two plane ellipse-shaped mirrors, having $12 \mathrm{~cm}$ as the projected diameter. The first mirror is built on a Newport RV80PP rotation stage, so that different elevation angles can be accessed. The whole setup with both mirrors is mounted on a Newport RV160PP rotation stage, so that desired azimuthal directions can be reached. This motor is able to move a considerable weight and is also carrying the "letterbox" shaped cover of the tracker so that the opening is always orientated into the observing direction. This cover can be opened, closed and sealed with pressurized air and was developed by the IMK in cooperation with Impres $\mathrm{GmbH}$ (http://www.impres.de). The RV160PP offers a hollow axle diameter of $11 \mathrm{~cm}$, through which the light falls vertically downwards into the container. The two motors are connected to a Newport XPS-Controller unit inside the container.

The optical setup inside the container is shown in Fig. 4. The optical path from the second tracking mirror to the input window of the spectrometer spans about $2.5 \mathrm{~m}$. The radiation is focused onto the input field stop of the spectrometer by an off-axis parabolic mirror. The field stop is an impor-

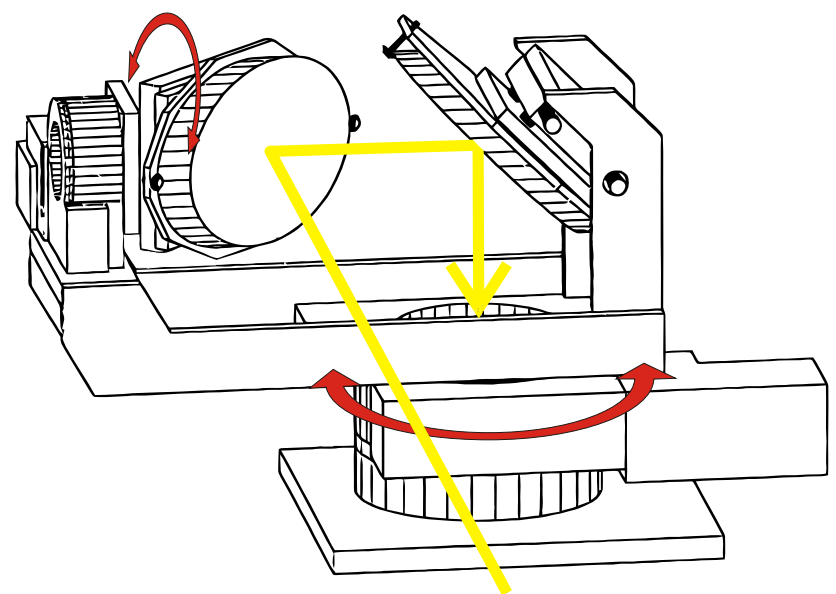

Fig. 3. Schematic drawing of the tracker used. (Picture taken from Huster, 1998.)

tant component of a high-resolution FTIR spectrometer as it defines the interferometer's field of view and so affects e.g. the instrumental line shape (Davis et al., 2001). It is realized by a flat metal wheel with round holes of different diameters, which can be rotated to select a desired field stop diameter. It is tilted a few degrees with respect to the light passing it to prevent reflections inside the spectrometer. The input field stop diameters used are smaller than the size of the picture of the solar disk on the field stop wheel of about $3.8 \mathrm{~mm}$, resulting from the focal length of the parabolic mirror $(418 \mathrm{~mm})$. After passing the field stop, the light is parallelized by a collimator and then enters the interferometer and finally is focused onto the detectors.

To monitor and control the pointing of our tracker, we use a standard CMOS USB-camera (VRmagic VRM C$9+$ PRO BW with $1280 \times 1024$ pixels) as an optical feedback, which records the radiation scattered back diffusely from the illuminated side of the field stop wheel. The distance between this camera and the field stop wheel is $25 \mathrm{~cm}$ and it is positioned in a way, that it does not affect the wanted signal used by the spectrometer. The camera is equipped with a standard objective and appropriate optical filters to adjust for the illumination level. Due to the wavelength-dependent refractivity of air, it is in case of NIR and MIR spectroscopic observations advantageous to equip the camera with an infrared longpass filter which transmits radiation beyond $750 \mathrm{~nm}$. In connection with the spectral sensitivity of current CCD and CMOS-cameras this choice defines a bandpass of about $100 \mathrm{~nm}$ width. (For further details on atmospheric dispersion see Sect. 7.) The solar disk has a diameter of about 240 pixels on the recorded pictures, the field stop diameters cover a range from about 30 to 160 pixels. This results in an angular size of about 8 arc s per pixel. The pictures are evaluated on a standard PC in real-time by our newly developed program using appropriate image processing algorithms, to determine the actual tracking accuracy and 


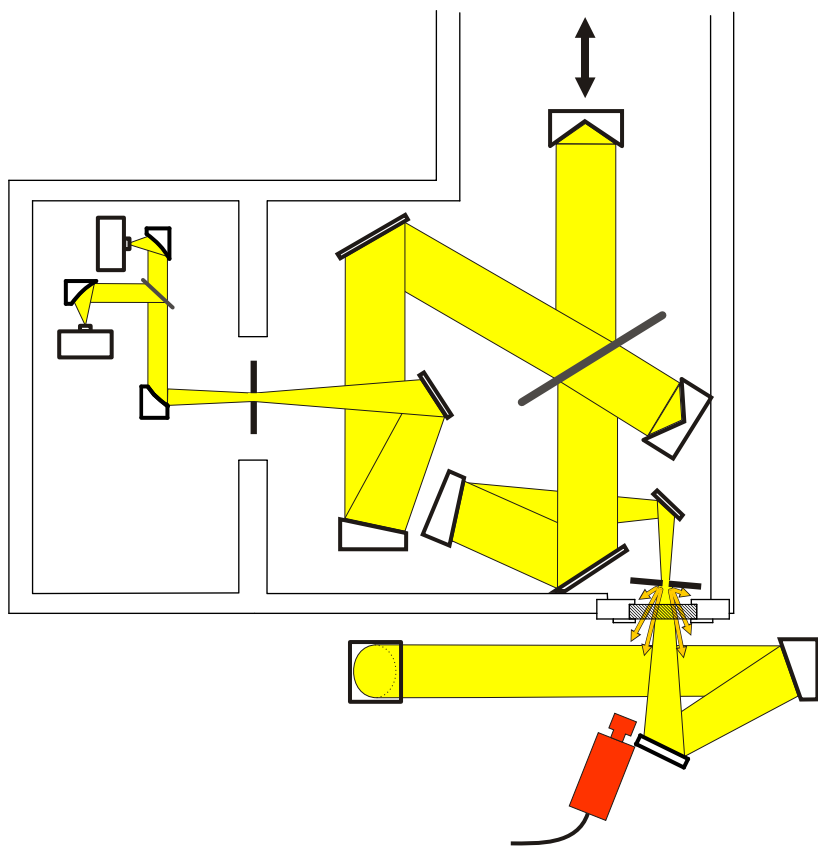

Fig. 4. Schematic drawing of the top-view of the camera set-up and the light path in front of and inside the spectrometer. The light falls from the tracker on the roof, perpendicular to the plane of the drawing onto the first mirror. The arrows indicate radiation scattered off by the aperture wheel, part of which is observed by the camera.

to calculate required corrections to the astronomical tracking mirror angles. The program then sends the trackingcommands to the motor-controller (Newport XPS) via an IPconnection.

\section{Principle of operation}

The operation principle of our tracker in Karlsruhe is based on a combination of astronomical algorithms to provide the coarse mirror angles with a superposition of small corrections to these angles derived from the optical feedback provided by the camera. The recorded pictures are evaluated by our software "CamTrack" in real-time, in order to determine both the central position of the field stop opening and the solar disk. Then the necessary mirror angle corrections to "move" the solar disk on the field stop wheel to the desired position relative to the input field stop opening are calculated and sent to the tracking system. These steps are continuously performed about three times per second. If no usable positions for the solar disk and the field stop opening can be determined, for example due to clouds, the system continues tracking on the basis of the astronomical coordinates together with previously saved offset values for similar tracking angles.

The main steps in determining the central positions from the camera pictures are the following:
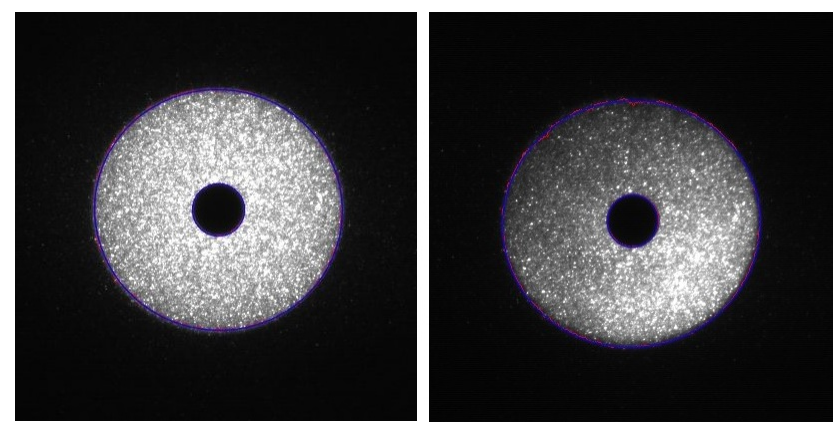

Fig. 5. Two pictures of the camera. The ellipses, which have been retrieved by the image processing algorithms are painted in blue on top of the original image. The solar disk and the field stop opening have diameters of 244 and 52 pixels respectively. The field stop diameter used was $0.8 \mathrm{~mm}$. The right picture shows a correct positioning of the solar disk relative to the field stop opening despite strong intensity variations resulting from clouds, which would be impossible using a quadrant diode.

1. Finding an appropriate threshold value to separate the bright area illuminated by the sun from the dark, nonilluminated rest of the field stop wheel, and the dark opening of the field stop.

2. Creating a binary (black/white) picture by applying the threshold, and finding the contours along the obtained areas (solar disk and field stop).

3. Fitting ellipses along the contours (in a least squares sense).

4. Performing consistency checks, if the obtained ellipses can be the contours of the solar disk and the field stop opening in terms of criteria like radius, position and eccentricity.

5. If the previous step was successful, taking the centers of the ellipses as centers of the solar disk and the round field stop opening.

Figure 5 shows image sections of two typical pictures of the camera. In cases where the field stop opening is not inside the solar disk, it is, in general, not visible. Then the solar disk is moved along a search pattern over the field stop wheel until the opening is found.

In order to calculate the corrections to the tracker mirror angles, one needs information on how the mirrors have to move, to realign the solar disk on the field stop. However, this correlation is not constant, but depends on the solar position on the sky. One way to get it, is to model the whole mirror system including the camera, which, however, is a quite complex task. A simple approach is to sequentially move the mirror angles a few small defined steps, and to register the resulting shift (direction and distance) of the solar disk in terms of camera pixels. This procedure cannot be performed during a FTIR measurement. Therefore we use a combination 
of the two procedures, which includes a simplified simulation of our two tracking mirrors only, and an experimentally determined correlation, at the beginning of the tracking, to initialize the simulation. This has the advantage that the effects of all the optical elements after the first two tracking mirrors are determined experimentally. For example, if the camera position or orientation changes or some additional mirrors are used, the only thing to do is to reperform the initialization sequence to adapt the simulation.

After this initialization procedure, the mirrors of the Camtracker setup are moved according to the calculated apparent motion of the solar disk. At a repeating rate of about $0.5 \mathrm{~s}$, images are recorded by the camera and the current position offset is determined using the algorithm described above. This correction is superimposed to the result of the astronomical calculation in the next commanding loop of the mirrors.

\section{Advantages of the camera setup}

The main reasons to choose the camera set-up instead of a quadrant-diode, which is the current solution applied in the NDACC and the TCCON, is that it results in a very exact tracking, it is easy to setup and very robust:

- Using the camera-information, one can determine both the position of the center of the solar disk on the field stop wheel and the opening of the stop at the same time. Since the input stop itself defines the measurement direction of the spectrometer one has direct information about the pointing and its errors. In other words, the camera-based optical feedback system is self-calibrating. Systematic shifts which can easily occur with a quadrant-diode setup are avoided. Displacements of the camera into any direction do not matter as long as the camera records a sufficiently focused picture of the solar disk and the field stop opening. This, as the only prerequisite, makes the system very easy to set-up and robust. Combined with an excellent spatial resolution of current cameras, this leads to a very precise tracking of the sun, as shown in Sect. 7.

- A very important advantage over a quadrant-diodesetup is the ability to precisely track light sources which have a variable intensity over their surface. For the sun, this can be caused by a rather thin cloud layer or mist which dims a part of the sun, as often happens at low solar angles. As long as one still can determine the rim of the solar disk, it is possible to determine the center of the disk and to maintain the precise line-of-sight (LOS) steering. An example can be seen in Fig. 5. A quadrantdiode, however, detects the center of intensity instead, which then is centered onto the input field stop.

- Resulting from the fact that the rim of the light source can be used instead of the center of intensity, the system provides the ability to track non-round light sources, such as the partially illuminated moon.

- By storing the pictures, even in the occurrence of tracking problems, one can determine the actual pointing directions later on and use them when evaluating the FTIR spectra. The stored pictures also offer a way to reveal the causes of unexpected intensity variations during subsequent inspections of the interferograms (e.g. moving objects such as clouds, birds, airplanes or transit of sun across fixed obstacles, e.g. contours of antennas, buildings, trees).

- The camera control loop is very easy to set up since no other optical elements except for the camera are needed. Its only prerequisite is the existence of an field stop which scatters some radiation back, so it is widely applicable.

\section{Tracking accuracies and results}

A first approach to determine the tracking accuracy of the Camtracker is to look at the distance between the centers of the two ellipses, which correspond to the solar disk and the field stop opening. This may not exactly be the actual accuracy of the pointing, since there may be deviations between the ellipse and the actual rim of the solar disk and residual errors resulting from small perspective distortions. Still we expect a useful quantification of the pointing error due to the motor resolution and mechanical backlash. Knowing the recorded solar disk diameter in camera pixels and its angular size on the sky of about 32 arc min, one can transform the ellipse-center distances in pixels to tracking-angle deviations in degrees. Figure 6 shows a plot of these deviations over a time period of more than $8 \mathrm{~h}$, from which a tracking accuracy of 3.7 arc s and a very small systematic error of about 0.3 arc s can be derived.

We also evaluated the solar Doppler line shifts for the spectra measured in Karlsruhe with the new camera setup in the NIR spectral domain, as described in Sect. 3. An example is shown in Fig. 7. To illustrate the sensitivity of the solar lineshift approach, we added a simulation for a $5 \operatorname{arc~min}$ tracking offset in the figure.

The solar shifts which were determined by this kind of analysis, are shown in Figs. 8 and 9. Assuming the tracking offsets to be of equal size along the direction perpendicular to the solar equator, the estimated tracking accuracy has a precision of about $\pm 11 \operatorname{arcs}(1 \sigma)$. The precision of the Doppler analysis has been crosschecked using a second microwindow (from 6248.3 to 6249.9 wavenumbers) and is about 2.5 arcs. An additional source of uncertainty in the analysis is the Doppler shift of the terrestrial reference lines due to wind, typically below 5 arc s (assuming wind speeds 

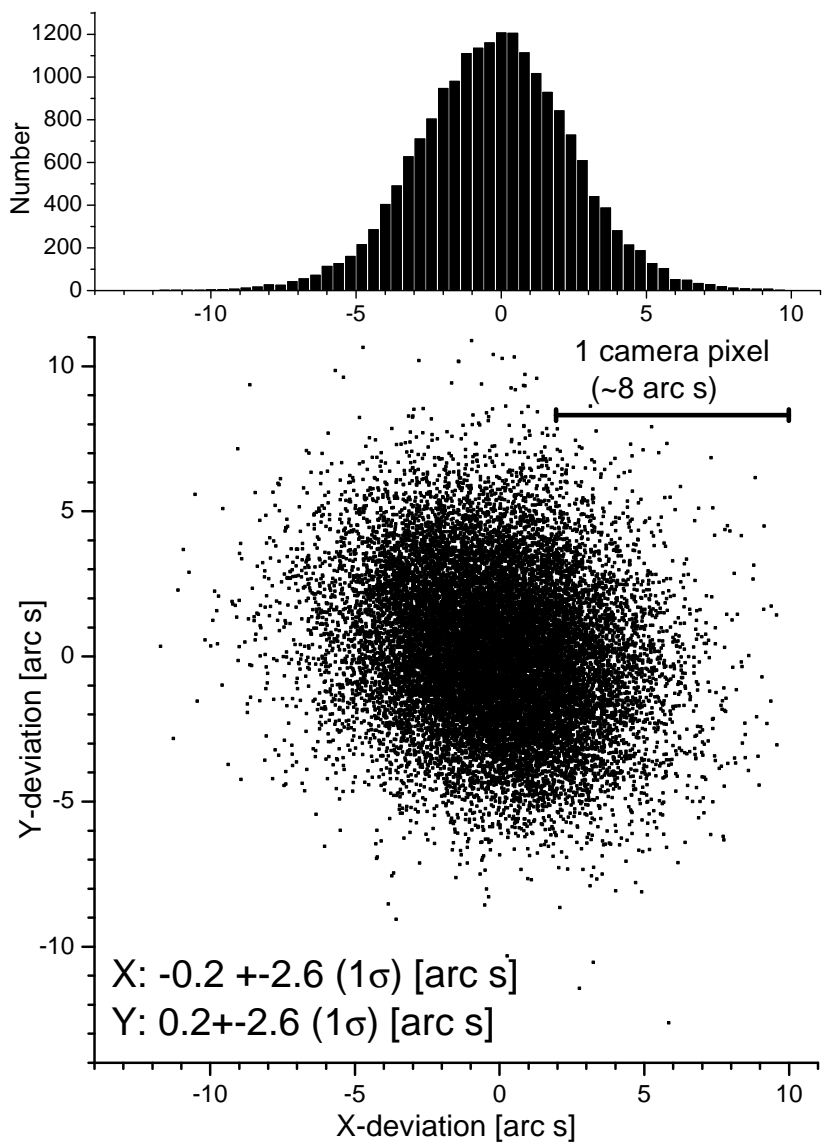

Fig. 6. Distance between the centers of the 2 fitted ellipses corresponding to the solar disk and the field stop opening as a 2$\mathrm{D}$ plot (lower graph) and the associated histogram along the $\mathrm{x}$ direction (upper graph). The units are given in tracking angle deviations. The data has a 2-D $1 \sigma$ interval of less than 4 arc s and was recorded on 22 September 2010, 10:29-16:43 UTC and 23 September 2010, 07:54-12:36 UTC, in two second intervals with a new version of "CamTrack". The previous version, used before 22 September 2010, had 2-D $1 \sigma$ interval of $6.5 \mathrm{arcs}$.

below $10 \mathrm{~m} \mathrm{~s}^{-1}$ ). The outstanding tracker precision is significantly better than the projected $19 \mathrm{arcs}$ and therefore sufficient to measure gas column concentrations with a precision below $0.1 \%$ for solar zenith angles smaller than $80^{\circ}$.

Before 22 September 2010, an older version of the "CamTrack" software was operating, showing an ellipse deviation of 6.5 arc s (compare Fig. 6). Therefore we expect the tracking accuracy to be even better than 11 arc s for current and future measurements. In the time series presented, the position and orientation of the camera changed frequently, due to modifications of our components in front of the FTIRspectrometer. This shows the robust mode of operation of the system and its independence from the position of the camera.

An overview of estimated contributions to the tracker pointing error budget is given in Table 1 for our current setup. To estimate the accuracy of the offset between the field stop

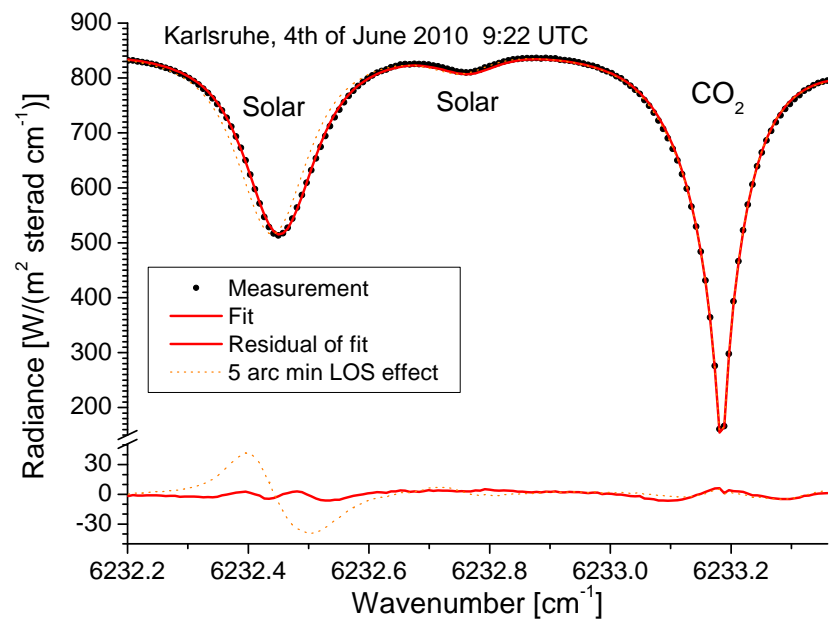

Fig. 7. Spectral microwindow to determine the solar shift for the spectra recorded in Karlsruhe. The black points correspond to the actual measurement, the red continuous lines to the fit and the residual. The dashed lines correspond to a simulation and for a pointing offset of 5 arc min along the solar equator.

Table 1. CamTracker pointing error contributions in arc $\mathrm{s}$.

\begin{tabular}{ll}
\hline Image reconstruction $^{1}$ & 2 \\
Atmospheric dispersion $^{2}$ & up to 3 at $80^{\circ} \mathrm{SZA}$ \\
Motor increment & 2.6 \\
Finite control loop duration $^{3}$ & $<2$ \\
Perspective distortion $^{3}$ & 2.4 \\
\hline
\end{tabular}

\footnotetext{
${ }^{1}$ Finite pixel size, sensor noise + image granularity.

${ }^{2}$ Can be removed by proper analysis procedure (see text).

${ }^{3}$ Will be further reduced in future software upgrade.
}

and the solar disc, we take into account three error sources. First, the theoretical accuracy to determine the center of an ideal circle $\sigma=\frac{0.42}{\sqrt{d}}$ (Haralick and Shapiro, 1993), where d is the diameter of an observed circular contour in units of pixels, assuming negligible obliquities of the ellipses. Two other sources limiting the accuracy are the noise generated by the image sensor and the granularity of the solar image. This granularity is due to the scattering properties of the matt finished aperture wheel surface (see Fig. 5). Other error contributions listed in the table are the atmospheric dispersion (discussed in Sect. 8), the finite angular resolution of the rotary stages, the finite duration of the control loop, and the perspective distortion which results from the fact that the camera records the aperture wheel with an angle of about $14^{\circ}$. This effect can be taken into account in the image analysis, but is not yet included in our software. 


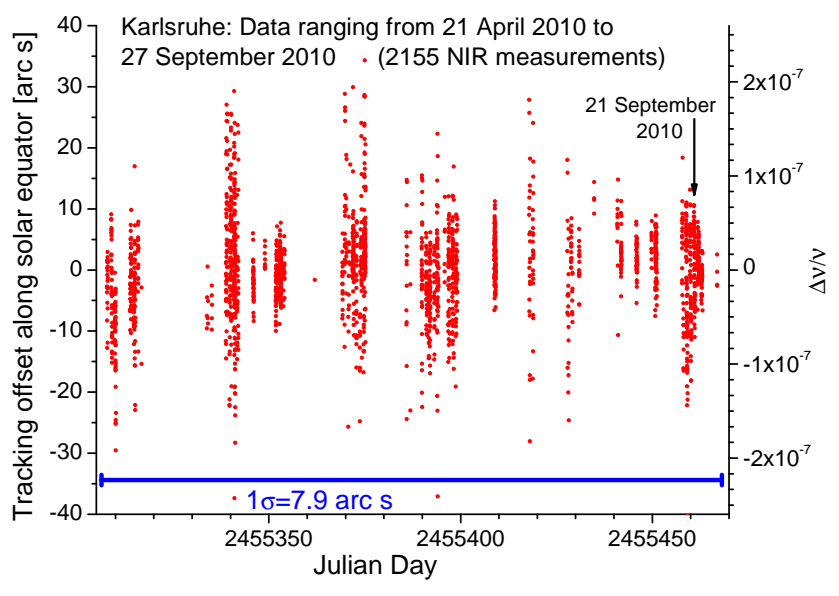

Fig. 8. Tracking angle offset along the solar equator determined by solar line shifts for 56 measurement days of observation at the Karlsruhe site. For both directions (2-D) a tracking accuracy of smaller than $\sqrt{2} \times \pm 7.9= \pm 11.2 \operatorname{arcs}(1 \sigma)$ can be estimated. The evaluated spectral window ranged from 6232.2 to 6233.36 wavenumbers (see Fig. 7).

\section{Implications for data analysis}

In order to take full advantage of the unprecedented precision which is achievable with the new Camtracker, we give a short discussion on implications for the data analysis in this section.

Firstly, it should be taken into account that the NIR/MIR ray path relevant for the quantitative analysis of the spectrum is not identical with the VIS/NIR ray path defined by the camera. Due to atmospheric refraction, the ray path is bent, its curvature being a function of pressure, $\mathrm{H}_{2} \mathrm{O}$ volume mixing ratio (VMR) and wavelength (Hase and Höpfner, 1999; Peck and Reeder, 1972; Jones, 1981; Matsumoto, 1982; Ciddor, 1996). The latter is the origin of atmospheric dispersion. In consequence the NIR/MIR solar disc image is slightly shifted versus the VIS/NIR solar disk images which is centered on the field stop by the camera control loop. Therefore, in the quantitative analysis of the spectrum, the raytracing should reproduce the apparent VIS/NIR apparent solar elevation angle at the observer, but use the refractive index appropriate for the IR bandpass of the spectrometer when tracing the raypath from the ground through the model atmosphere. Quantitatively, at $80^{\circ} \mathrm{SZA}$, the total deviation of the beam due to refraction is in the order of $300 \mathrm{arcs}$, a $750 \mathrm{~nm}$ ray being deviated by an additional 3 arc s compared to a $2 \mu \mathrm{m}$ ray.

The second aspect is the temporal extent of the FTIR measurement. Depending on the spectral resolution applied, the recording of an interferogram requires up to several minutes. Current analysis schemes for ground-based FTIR-spectra assume a single pencil-beam LOS, whereas the measurement covers a finite range of elevation angles. A detailed discus-

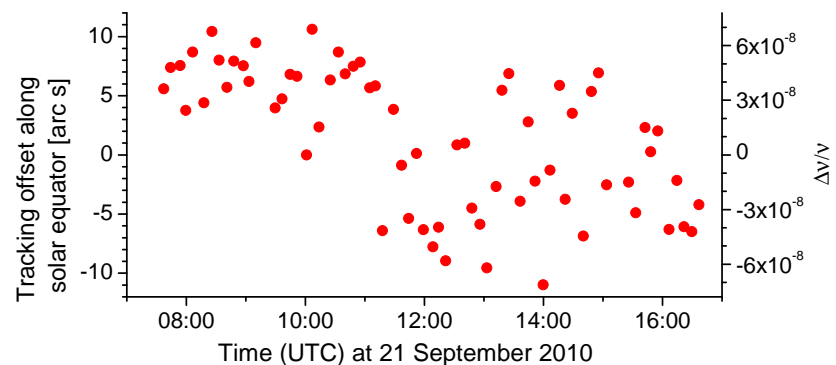

Fig. 9. Enlarged part of Fig. 8 for one sample measuremet day (21 September 2010).

sion of this problem is beyond the scope of this technical paper, which deals with the Camtracker setup and precision verification. The optimum choice of an effective solar elevation angle and the allowable measurement duration as function of latitude and solar elevation will be addressed in a subsequent paper dealing with the impact of the variable solar elevation from the viewpoint of the analysis.

\section{Conclusions}

The presented camera set-up in combination with the realtime image evaluation and tracking software "CamTrack" was shown to result in an outstanding tracking accuracy of better than 11 arc s. This quality level was maintained over a period of 5 months, despite frequent changes of the camera position, showing the robustness of the operation principle. The system is easy to setup and applicable in many situations, where there is the need to position the radiation of a light source on the field stop of a spectrometer.

Acknowledgements. We would like to thank the NASA Goddard Space Flight Center for providing NCEP daily temperature and pressure profiles (via the automailer system) which were used for the Doppler analysis and for the calculation of the solar refraction.

Edited by: J. Notholt

\section{References}

Abrams, M. C., Toon, G. C., and Schindler, R. A.: Practical example of the correction of Fourier-transform spectra for detector nonlinearity, Appl. Optics, 33(27), 6307-6314, doi:10.1364/AO.33.006307, 1994.

Adrian, G. P., Baumann, M., Blumenstock, T., Fischer, H., Friedle, A., Gerhardt, L., Maucher, G., Oelhaf, H., Scheuerpflug, W., Thomas, P., Trieschmann, O., and Wegner, A.: First results of ground-based FTIR measurements of atmospheric trace gases in North Sweden and Greenland during EASOE, Geophys. Res. Lett., 21, 1343-1346, 1994.

Ciddor, P. E.: Refractive index of air: new equations for the visible and near infrared, Appl. Optics, 35, 1566-1573, 1996. 
Davis, S. D., Abrams, M. C., and Brault, J. W.: Fourier Transform Spectrometry, Academic Press, 2010.

Haralick, R. M. and Shapiro, L. G.: Computer and robot vision, Edition 1, Volume 2, Addison-Wesley publishing company, 535542, 1993.

Hase, F.: Inversion von Spurengasprofilen aus hochaufgelösten bodengebundenen FTIR-Messungen in Absorption, Dissertation, FZK Report No. 6512, http://www-imk.fzk.de/asf/ftir/disshase. pdf, last access: 28 October 2010, Forschungszentrum Karlsruhe, Germany, 2000.

Hase, F. and Höpfner, M.: Atmospheric raypath modelling for radiative transfer algorithms, Appl. Optics, 38, 3129-3133, 1999.

Hase, F., Blumenstock, T., and Paton-Walsh, C.: Analysis of the instrumental line shape of high-resolution Fourier transform IR spectrometers with gas cell measurements and new retrieval software, Appl. Optics, 38, 3417-3422, 1999.

Hase, F., Hannigan, J. W., Coffey, M. T., Goldman, A., Höpfner, M., Jones, N. B., Rinsland, C. P., and Wood, S. W.: Intercomparison of retrieval codes used for the analysis of high-resolution, ground-based FTIR measurements, J. Quant. Spectrosc. Ra., 87, 25-52, 2004.

Hase, F., Demoulin, P., Sauval, A. J., Toon, G. C., Bernath, P. F., Goldman, A., Hannigan, J. W., and Rinsland, C. P.: An empirical line-by-line model for the infrared solar transmittance spectrum from 700 to $5000 \mathrm{~cm}^{-1}$, J. Quant. Spectrosc. Ra., 102, 450-463, doi:10.1016/j.jqsrt.2006.02.026, 2006.

Hase, F., Wallace, L., McLeod, S. D., Harrison, J. J., and Bernath, P. F.: The ACE-FTS atlas of the infrared solar spectrum, J. Quant. Spectrosc. Ra., 111(4), 521-528, 2010.

Huster, S.: Bau eines automatischen Sonnenverfolgers fr bodengebundene IR-Absorptionsmessungen, Diploma Thesis, Forschungszentrum Karlruhe, Universität Karlsruhe, 1998.

Jones, F. E.: The refractivity of air, J. Res. Nat. Bur. Stand., 86, 27-32, 1981.

Keppel-Aleks, G., Toon, G. C., Wennberg, P. O., and Deutscher, N. M.: Reducing the Impact of Source Brightness Fluctuations on spectra obtained by FTS, Appl. Optics, 46, 4774-4779, 2007.

Lang, K. R.: Astrophysical Data: Planets and Stars, Springer Verlag, ISBN 0-387-97109-2, 1991.

Matsumoto, H.: The Refractive Index of Moist Air in the 3- $\mu \mathrm{m}$ Region, Metrologia, 18, 49-52, doi:10.1088/0026-1394/18/2/001, 1982.
Messerschmidt, J., Macatangay, R., Notholt, J., Petri, C., Warneke, T., and Weinzierl, C.: Side by side measurements of $\mathrm{CO}_{2}$ by ground-based Fourier transform spectrometry (FTS), Tellus B, 62, 749-758, 2010.

Notholt, J. and Schrems, O.: Shipborne FT-IR Measurements of Atmospheric Trace Gases on a South $\left(33^{\circ} \mathrm{S}\right)$ to North $\left(53^{\circ} \mathrm{N}\right)$ Atlantic Traverse, Appl. Spectrosc., 49, 10, 1525-1527, 1995.

Olsen, S. C. and Randerson, J. T.:, Differences between surface and column atmospheric $\mathrm{CO}_{2}$ and implications for carbon cycle research, J. Geophys. Res., 109, D02301, doi:10.1029/2003JD003968, 2004.

Peck, E. R. and Reeder, K.: Dispersion of air, J. Opt. Soc. Am., 62(8), 958-962, doi:10.1364/JOSA.62.000958, 1972.

Toon, G., Blavier, J.-F., Washenfelder, R., Wunch, D., KeppelAleks, G., Wennberg, P., Connor, B., Sherlock, V., Griffith, D. Deutscher, N., and Notholt, J.: Total Column Carbon Observing Network (TCCON), http://www.tccon.caltech.edu/publications/ OSA_FTS_Meeting_20090323.pdf, last access: 28 October 2010, 2009.

Washenfelder, R. A., Toon, G. C., Blavier, J.-F., Yang, Z., Allen, N. T., Wennberg, P. O., Vay, S. A., Matross, D. M., and Daube, B. C.: Carbon dioxide column abundances at the Wisconsin Tall Tower site, J. Geophys. Res., 111, D22305, doi:10.1029/2006JD007154, 2006.

Wunch, D., Toon, G. C., Wennberg, P. O., Wofsy, S. C., Stephens, B. B., Fischer, M. L., Uchino, O., Abshire, J. B., Bernath, P., Biraud, S. C., Blavier, J.-F. L., Boone, C., Bowman, K. P., Browell, E. V., Campos, T., Connor, B. J., Daube, B. C., Deutscher, N. M., Diao, M., Elkins, J. W., Gerbig, C., Gottlieb, E., Griffith, D. W. T., Hurst, D. F., Jiménez, R., Keppel-Aleks, G., Kort, E. A., Macatangay, R., Machida, T., Matsueda, H., Moore, F., Morino, I., Park, S., Robinson, J., Roehl, C. M., Sawa, Y., Sherlock, V., Sweeney, C., Tanaka, T., and Zondlo, M. A.: Calibration of the Total Carbon Column Observing Network using aircraft profile data, Atmos. Meas. Tech., 3, 1351-1362, doi:10.5194/amt-31351-2010, 2010a.

Wunch, D., Toon, G., Blavier, J.-F. L., Washenfelder, R. A., Notholt, J., Connor, B. J., Griffith, D. W. T., Sherlock, V., and Wennberg, P. O.: The Total Carbon Column Observing Network (TCCON), Philos. T. R. Soc. A, in press, 2010b. 